\title{
A Hybrid Approach for Pap-Smear Cell Nucleus Extraction
}

\author{
M. Orozco-Monteagudo ${ }^{1}$, Hichem Sahli ${ }^{2}$, Cosmin Mihai ${ }^{2}$, \\ and A. Taboada-Crispi ${ }^{1}$ \\ 1 Universidad Central de Las Villas, Cuba \\ morozco@uclv.edu.cu, ataboada@uclv.edu.cu \\ 2 Vrije Universiteit Brussel, Electronics and Informatics Dept. - ETRO, \\ Pleinlaan 2, 1050 Brussels, Belgium \\ hsahli@etro.vub.ac.be, cmihai@etro.vub.ac.be
}

\begin{abstract}
This paper, proposes a two-phases approach for a computerassisted screening system that aims at early diagnosis of cervical cancer in Pap smear images and accurate segmentation of nuclei. The first phase uses spectral, shape as well as the class membership to produce a nested hierarchical partition (hierarchy of segmentations). The second phase, selects the best hierarchical level based on an unsupervised criterion, and refines the obtained segmentation by classifying the individual regions using a Support Vector Machine (SVM) classifier followed by merging adjacent regions belonging to the same class. The effectiveness of the proposed approach for producing a better separation of nucleus regions and cytoplasm areas is demonstrated using both ground truth data, being manually segmented images by pathologist experts, and comparison with state-of-art methods.
\end{abstract}

Keywords: microscopic images, cell segmentation, watershed, SVM classification.

\section{Introduction}

Cervical cancer, currently associated with the Human Papilloma Virus as one of the major risk factors, affects thousands of women each year. The Papanicolaou test (known as Pap test) is used to detect pre-malignant and malignant changes in the cervix [1]. Cervical cancer can be mostly prevented by early detection of abnormal cells in smear tests.

Due to the cervix is wiped out with a swap, Pap test is classified as an invasive method. It is only used for screening purposes and not for diagnosis. These cells are examined under a microscope for abnormalities. Trained biologist are required to evaluate these tests. In underdeveloped countries, the death rate due to cervical cancer is significantly higher due to the lack of personnel trained in this field and repeated follow-up tests. As a result, women in developed countries have less than $0.1 \%$ chance of developing cervical cancer while their counterparts in underdeveloped countries have a $3-5 \%$ chance of developing cervical cancer. 


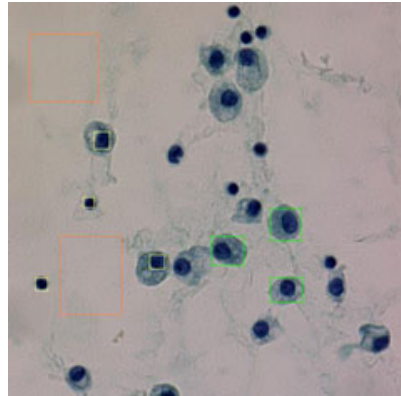

(a)

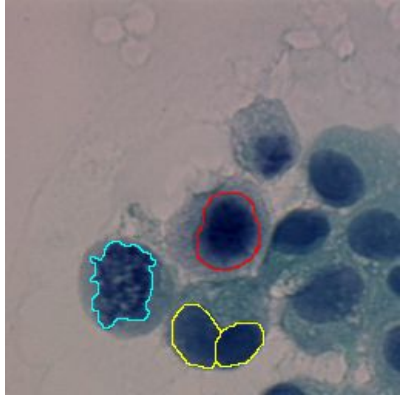

(b)

Fig. 1. Pap-smear cell images. (a) Dark blue parts (yellow rectangle) represents the nuclei. Pale blue part (green rectangle) are the cytoplasms. Magenta parts (orange rectangle) are the background. (b) Nucleus Variability.

As illustrated in Fig. 1, two classes of regions are considered: nucleus regions and other regions that include cytoplasm and background. The overall proportion of the nucleus pixels is approximately between $7 \%$ and $10 \%$. Cells nucleus are blue (dark to pale) and cytoplasms are blue-green (Fig. 17). Red blood corpuscles are coloured reddish. The spatial configuration and the colour of the cells is extremely variable (Fig. 10). Isolated or touching cells as well as clustered or overlapping cells can be found.

The automated segmentation of cell nuclei in Pap smear images is one of the most interesting fields in cytological image analysis [2]. In the last years, cell nucleus segmentation has been extensively studied by several researchers, in [3, frequency domain features are used to detect abnormal cervical cell images. In [4], statistical geometric features, which are computed from several binary thresholded versions of texture images are used to classify among normal and abnormal cervical cells. Lezoray et al. [5] extract the nuclei of the cervical cells using a combination of a colour pixel classification scheme $(k$-means and Bayesian classification) with a colour watershed segmentation algorithm. In 6] a segmentation scheme and its performance is evaluated using Pap-smear samples in the presence of heavy additive noise.

Developing automated algorithms for segmenting nuclei continue to pose interesting challenges. Much of the difficulty arises from the inherent colour and shape variability. The goal of the present work is to develop automated and computationally efficient algorithms that improve upon previous methods using watershed approach. In this work, we propose a hybrid two-steps approach to cell segmentation in Pap smear images. The first phase consists of creating a nested hierarchy of partitions, which produces a hierarchical segmentation that uses the spectral, shape information as well as the class information. The most meaningful hierarchical level is then detected using a segmentation quality criterion. The second phase aims at identifying the nucleus and cytoplasm 
areas by classifying the segments (regions) resulting from the first phase using multiple spectral and shape features, and further merging the neighboring regions belonging to the same class. The selection of individual regions is obtained using a SVM classifier, based on spectral and shape features.

The reminder of the paper is organized as follows. Section 2 describes the segmentation algorithm used to segment the images and produces a hierarchy of nested partitions. Section 3.1 proposes an unsupervised segmentation quality criterion to select a hierarchical level on which an SVM classification is applied in Section 3.2 to classify the segmented region in nucleus/non-nucleus and pruning the segmentation errors by merging adjacent segmented regions which may have been over-segmented. Section 4 presents and discusses the obtained results. Finally, conclusions are presented in Section 5 .

\section{Hierarchy of Partitions of Pap-Smear Cell Images}

The waterfall algorithm [7] is used here for producing a nested hierarchy of partitions, $\mathcal{P}^{h}=\left\{r_{1}^{h}, r_{2}^{h}, \ldots, r_{m_{h}}^{h}\right\} ; h=1, \cdots n$, which preserves the inclusion relationship $\mathcal{P}^{h} \supseteq \mathcal{P}^{h-1}$, implying that each atom of the set $\mathcal{P}^{h}$ is a disjoint union of atoms from the set $\mathcal{P}^{h-1}$. For successively creating hierarchical partitions, the waterfall algorithm removes from the current partition (hierarchical level) all the boundaries completely surrounded by higher boundaries. The staring partition, is obtained using watershed transform [8], being a morphological segmentation applied on the gradient magnitude of an image in order to guide the watershed lines to follow the crest lines and the real boundaries of the regions. In our implementation, we use the DiZenzo gradient [9], which calculates the maximum rate of change in one pixel based in partial derivatives in $R G B$ colour space.

For producing the nested hierarchy, in this work, we use the approach proposed in [10], where the saliency measure, $E\left(\tilde{r}=r_{i} \cup r_{j} \mid r_{i}, r_{j}\right)$, of a boundary between two neighboring segments $r_{i}$ and $r_{j}$ (being the cost of merging the regions $r_{i}$ and $r_{j}$ ), is based on two energy functions used to characterize desired single-segment properties, and pair-wise segment properties [10]:

$$
E\left(\tilde{r}=r_{i} \cup r_{j} \mid r_{i}, r_{j}\right)=E(\tilde{r})+E\left(r_{i}, r_{j}\right)
$$

The single-segment properties, $E(\tilde{r})$, is the merged region property as defined in [10, it includes segment homogeneity $\left(E_{\text {hom }}\right)$, segment convexity $\left(E_{\text {conv }}\right)$, segment compactness $\left(E_{\text {comp }}\right)$, and colour variances $\left(E_{\operatorname{var}_{c}}\right)$ within the segment:

$$
\begin{aligned}
E(\tilde{r}) & =\frac{1}{E_{\mathrm{hom}}(\tilde{r})} \cdot \sum_{c} E_{\mathrm{var}_{c}}(\tilde{r}) . \\
& \left(1+\left|E_{\mathrm{conv}}(\tilde{r})\right|\right)^{\operatorname{sign}\left(E_{\mathrm{conv}}(\tilde{r})\right)} \cdot\left(1+\left|E_{\mathrm{comp}}(\tilde{r})\right|\right)^{\operatorname{sign}\left(E_{\mathrm{comp}}(\tilde{r})\right)} .
\end{aligned}
$$

The pair-wise property, $E\left(r_{i}, r_{j}\right)$, as defined in [10] includes the dynamics of the contour and the color difference between the neighboring regions. 
In this work, considering the type of images we are dealing with, we propose the following merging criterion:

$$
E\left(\tilde{r}=r_{i} \cup r_{j} \mid r_{i}, r_{j}\right)=\phi\left(c_{i}=c_{j} \mid r_{i}, r_{j}\right) \cdot\left(E(\tilde{r})+E\left(r_{i}, r_{j}\right)\right)
$$

where, $\phi\left(c_{i}=c_{j} \mid r_{i}, r_{j}\right)$ is a factor favoring the merging of regions with similar classes [11] $E\left(r_{i}, r_{j}\right)$, the pair-wise region property, defined as:

$$
E\left(r_{i}, r_{j}\right)=-\log \left(\sum_{k=1}^{b} \sqrt{P_{r_{i}}^{(k)} \cdot P_{r_{j}}^{(k)}}\right) .
$$

being the Bhattacharyya merging criterion proposed in [12], with the number of bins used $b=32$.

Different from 11, the parameter $\phi\left(c_{i}=c_{j} \mid r_{i}, r_{j}\right)$, representing the potential of merging neighboring regions with similar class membership, is here defined as follows:

$$
\phi\left(c_{i}=c_{j} \mid r_{i}, r_{j}\right)=\frac{1}{1+\operatorname{Pr}\left(c_{i}=c_{j} \mid \mathbf{f}\left(r_{i}\right), \mathbf{f}\left(r_{j}\right)\right)} .
$$

where $c_{i}, c_{j} \in \Omega=\left\{\omega_{1}=\right.$ nucleus, $\omega_{2}=$ no-nucleus $\}$, are the classes for $r_{i}$ and $r_{j}$, respectively, and $\operatorname{Pr}\left(c_{i}=c_{j} \mid \mathbf{f}\left(r_{i}\right), \mathbf{f}\left(r_{j}\right)\right)$ is the probability that $r_{i}$ and $r_{j}$ belong to the same class, given the feature vectors $\mathbf{f}\left(r_{i}\right)$ and $\mathbf{f}\left(r_{j}\right)$. In our approach, $\operatorname{Pr}\left(c_{i}=c_{j} \mid \mathbf{f}\left(r_{i}\right), \mathbf{f}\left(r_{j}\right)\right)$ is calculated using the method of Platt 13. from the output of a two classes SVM [14 trained using as feature vector, $\mathbf{f}(r)=\left[\mu(r(L)), \mu\left(r(a), \mu(r(b)]^{t}\right.\right.$, consisting of the mean of the $L, a$, and $b$ channels of the region $r$ in the $L a b$ colour space.

The parameters of the SVM classifier have been selected as follows. A linear kernel SVM and gaussian kernel SVMs (with different values for $\sigma$ ) were trained using a 10-fold cross-validation. A grid search method was used to select the best parameters of the SVM. The penalty parameter of the error $C$ was tested in $C=\left\{2^{i}: i=-1 . .14, \infty\right\}$, as well as the parameter of the gaussian kernel $\sigma$ in $\sigma=\left\{2^{i}: i=-3 . .4\right\}$. The best performance was obtained for $C=1024$ and gaussian kernel SVM with $\sigma=0.5$. Finally,

$$
\operatorname{Pr}\left(c_{i}=c_{j} \mid \mathbf{f}\left(r_{i}\right), \mathbf{f}\left(r_{j}\right)\right)=p_{1} p_{2}+\left(1-p_{1}\right)\left(1-p_{2}\right)
$$

with $p_{1}=\operatorname{Pr}\left(c_{i}=\omega_{1} \mid f\right)$ and $p_{2}=\operatorname{Pr}\left(c_{j}=\omega_{1} \mid f\right)$, are estimated using the method of Platt 13 which adjusts the output of a SVM by using a sigmoid function

$$
\operatorname{Pr}\left(\operatorname{class}=\omega_{k} \mid f\right)=\frac{1}{1+\exp (A f+B)} .
$$

where $f$ is the output of the SVM, and the parameters $A$ and $B$ are fitted using maximum likelihood estimation. 


\section{Pap-Smear Cell Images Segmentation and Classification}

\subsection{Segmentation Level Selection}

As mentioned above, the output of the hierarchical segmentation is a set of partitions $\mathcal{P}^{h}=\left\{r_{1}^{h}, r_{2}^{h}, \ldots, r_{m_{h}}^{h}\right\} ; h=1, \cdots n$. In order to select the best segmentation level, for further analysis, in this work we use the criterion of Borsotti et al. [15]:

$$
\begin{aligned}
\operatorname{BOR}\left(\mathcal{P}^{h}\right)= & 1-\frac{\sqrt{m_{h}}}{10^{4} \cdot \operatorname{Card}(I)} . \\
& \sum_{k=1}^{m_{h}}\left[\frac{E_{k}^{2}}{1+\log \left(\operatorname{Card}\left(r_{k}^{h}\right)\right)}+\left(\frac{\chi\left(\operatorname{Card}\left(r_{k}^{h}\right)\right)}{\operatorname{Card}\left(r_{k}^{h}\right)}\right)^{2}\right] .
\end{aligned}
$$

where, $\operatorname{Card}()$ is the size (area) or a region $r_{k}^{h}$ or the image $I ; \chi\left(\operatorname{Card}\left(r_{k}^{h}\right)\right)$ is the number of regions having the same size (area) as region $r_{k}^{h}$; and $E_{k}$ is sum of the Euclidean distances between the RGB colour vector of the pixels of $r_{k}$ and the colour vector attributed to the region $r_{k}$ in the segmentation result. This criterion allows penalizing both over-segmentation (small regions) and undersegmentation (regions that have a large color error).

The best segmentation level is the one which produces the maximum value of the BOR criterion Eq (8).

\subsection{SVM Region Classification and Merging}

The Borsotti et al. [15] criterion Eq (8) is a good unsupervised segmentation quality measure, however most of the time the best value does not correspond to the best segmentation level according to the biologists criteria (Fig. 2(2) versus Fig 2(6)). A suitable approach is to prune the segmentation, resulting from the hierarchical level selection criterion, by merging adjacent regions belonging to the same class. Indeed, as depicted in Fig. 2, the selected level shows a cell with 2 regions, after region-based classification and extra merging, the final segmentation/classification results has been refined.

Support vector machines (SVM) have been proven to be powerful and robust tools for tackling classification tasks [14]. Different from mostly used SVM pixelbased classification, we propose to apply SVM on region-based features and classify the segments of the selected level into nucleus and non-nucleus regions. A set of 116 region features were first calculated. In an attempt to optimize the dimensionality of the feature set, a subset of features was selected via stepwise discriminant analysis [16. This method uses Wilks' $\lambda$ statistic to iteratively determine which features are best able to separate the classes from one another in the feature space. Since it is not possible to identify a subset of features that are optimal for classification without training and testing classifiers for all combinations of the input features, optimization of Wilks' $\lambda$ is a good choice.

Table 1, lists the identified nine (out of 116) features that were the most statistically significant in terms of their ability to separate the two considered classes, nucleus and no nucleus regions (cytoplasm and background). 
Table 1. Selected features using stepwise discriminant analysis

\begin{tabular}{l}
\hline$F_{1}$. Mean of the green channel \\
$F_{2} .0 .1-$ trimmean of the blue channel \\
$F_{3}$. Solidity \\
$F_{4}$. Max value of the red channel \\
$F_{5}$. Edge fraction of pixels along the edges \\
$F_{6}$. Edge gradient intensity homogeneity \\
$F_{7}$. Edge direction difference. \\
$F_{8}$. Shape factor of the convex hull \\
$F_{9}$. Region area \\
\hline
\end{tabular}

\section{Results and Discussion}

Fig. 3 illustrates the proposed approach in one of the tested images. The first row depicts some hierarchical levels along with their BOR criterion and number of regions. As it can be noticed, the hierarchical Level-1 is the best according to the BOR criterion. Moreover, the BOR criterion between the first three levels is almost identical. After SVM classification and the merging of neighboring regions belonging to the same class (second and third rows of Figure 3), the hierarchical Level-2 gives better segmentation results with respect to the BOR criterion, and the Vinet distance, $V$, [5]. This is also confirmed by classification results as shown in Table 2 .

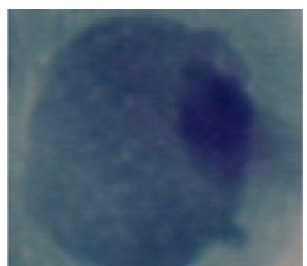

(1)

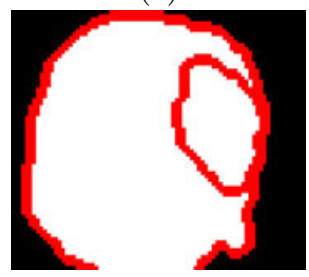

(4)

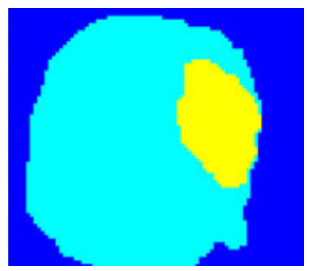

$(2)$

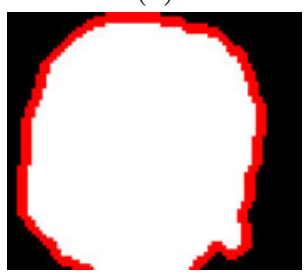

(5)



(3)

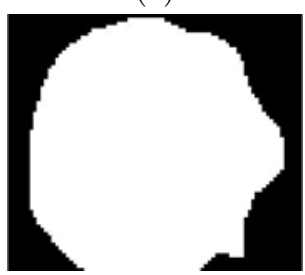

(6)

Fig. 2. Merging after classification. (1) Original image. (2) Hierarchical Level Selection Results: Labeled image. (3) Mosaic image. (4) Region Classification Results (white mean nucleus). (5) Merging of regions that belong to the same class. (6) Manually delineated nucleus. 


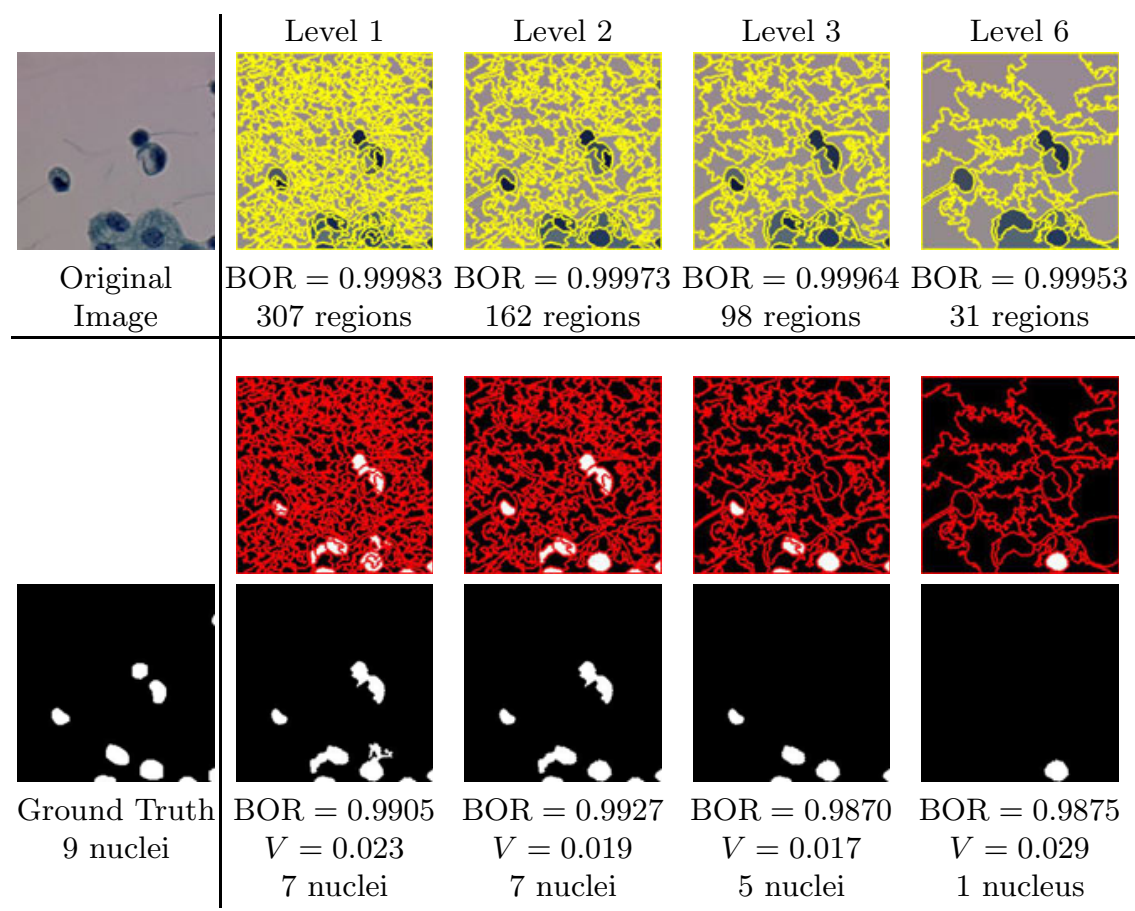

Fig. 3. Illustration of the approach. (Upper-Left) Original Image. (Bottom-Left) Ground Truth. (Upper-Right) Four Hierarchical Levels. (Bottom-Right) Results after classification and second merging.

Table 2. Confusion matrices - SVM classification of the regions shown in Fig. 3

\begin{tabular}{llll}
\hline Level & Nucleus & Non-Nucleus \\
\hline \multirow{2}{*}{$\mathbf{1}$} & Nucleus & $290(100.0 \%)$ & $0(0.00 \%)$ \\
& Non-Nucleus $4(23.53 \%)$ & $13(76.47 \%)$ \\
\hline \multirow{2}{*}{$\mathbf{2}$} & Nucleus & $151(100.0 \%)$ & $0(0.00 \%)$ \\
& Non-Nucleus 1 $1(9.09 \%)$ & $10(90.91 \%)$ \\
\hline \multirow{2}{*}{$\mathbf{3}$} & Nucleus & $91(98.91 \%)$ & $1(1.09 \%)$ \\
& Non-Nucleus 0 $(0.00 \%)$ & $6(100.0 \%)$ \\
\hline \multirow{2}{*}{$\mathbf{l}$} & Nucleus & $29(96.67 \%)$ & $1(3.33 \%)$ \\
& Non-Nucleus 0 $(0.00 \%)$ & $1(100.0 \%)$ \\
\hline
\end{tabular}

The Vinet distance is a widely used measure to quantify the difference between two segmentations (one of them is frequently a ground truth). For an image $I$ of $N$ pixels and two segmentations $A$ and $B$, with $m$ and $n$ regions, respectively. First, a label superposition table is computed: $T_{i j}=\left|A_{i} \cup B_{j}\right|$ with $0 \leq i \leq m$ and $0 \leq j \leq n$. The maximum of this matrix gives the two most similar regions extracted from $A$ and $B$, respectively. The similarity criterion is defined by: 
$C_{0}=\max \left(T_{i j}\right)$ with $0 \leq i \leq m$ and $0 \leq j \leq n$. The search of the second maximum (without taking into account the two last regions) gives the similarity criterion $C_{1}$ and so on to $C_{k-1}$, where $k=\min (m, n)$. The dissimilarity measure between the two segmentations $A$ and $B$ is given by:

$$
D(A, B)=1-\frac{1}{N} \cdot \sum_{i=0}^{k-1} C_{i} .
$$

The proposed approach was applied to twenty images that contains approximately 160 nuclei. The training of the SVM was made using the SVM-KM toolbox [17. The evaluation of the proposed approach was made using a leave-one-out cross-validation using two different criteria:

- Segmentation quality: using the Vinet distance 5] according to a manually extracted ground truth.

- Classification quality: using the Accuracy and F-measures [18] according to a manually extracted ground truth.

Table 3. Overall Assesment

\begin{tabular}{lccc}
\hline SVM Classifier & Vinet Measure Accuracy & F-measure \\
\hline Linear Kernel & 0.0223 & 0.9733 & 0.9853 \\
Gaussian Kernel $\sigma=0.5$ & 0.0494 & 0.9109 & 0.9587 \\
Gaussian Kernel $\sigma=1$ & 0.0456 & 0.9235 & 0.9644 \\
Gaussian Kernel $\sigma=2$ & 0.0407 & 0.9448 & 0.9704 \\
Gaussian Kernel $\sigma=4$ & 0.0323 & 0.9592 & 0.9781 \\
Gaussian Kernel $\sigma=8$ & 0.0274 & 0.9668 & 0.9821 \\
Linear Kernel $d=2$ & 0.0375 & 0.9546 & 0.9750 \\
Linear Kernel $d=3$ & 0.0528 & 0.9552 & 0.9758 \\
\hline CCW & 0.0455 & 0.9571 & 0.6445 \\
GEE & 0.0243 & 0.9784 & 0.9880 \\
SVMP & 0.0356 & 0.9743 & 0.8601 \\
\hline
\end{tabular}

Table 3 summarizes the average of the Vinet measure, Accuracy, and F-measures, for all the testing images, versus SVM kernels. As it can be seen the best results are obtained using SVM classifier with a linear kernel. The Confusion matrix using a linear kernel is given in Table 4.

Table 4. Confusion Matrix for SVM classifier with a linear kernel

\begin{tabular}{lrr}
\hline & Nucleus & Non-Nucleus \\
\hline Nucleus & $98.49 \%$ & $1.51 \%$ \\
Non-Nucleus & $9.16 \%$ & $90.84 \%$ \\
\hline
\end{tabular}

To further assess our results, we give in the last rows of Table 3 the results obtained using three state of art methods, namely, the Cooperative colour watershed proposed in [5] (CCW), the Hierarchical Segmentation of [10] (GEE), and 
a Pixel-based SVM classification (SVMP) [14. As it can be seen from Table 3, the proposed approach produces good results.

\section{Conclusions}

In this work, we introduced a hybrid segmentation/classification approach which improves the automatic segmentation of nuclei for the purpose of the Papanicolaou test. First, a classification factor was introduced during the process of merging neighboring segments during the hierarchical segmentation process. Second we introduced a non supervised approach for the selection of the best hierarchical segmentation level. Finally, to prune most of the wrongly segmented cells and avoid over/under segmentation, we introduced a region-based SVM classifier able of improving the performance of the resulting segmentation. The SVM classifier was used to separate the two classes of regions: nucleus and no nucleus regions (cytoplasm and background) using an appropriate set of region features (morphometrics, edge-based, and convex hull-based). Our method is adapted to the segmentation of cellular objects. A leave-one-out cross-validation approach allowed proving that the proposed approach produces a segmentation closer to what is expected by human experts. In order to improve the segmentation results (separating cells) we will consider applying vector image restoration based on Partial Differential Equations (PDE) [19.

\section{Acknowledgement}

This work was partially supported by the Canadian International Development Agency Project Tier II-394-TT02-00 and by the Flemish VLIR-UOS Programme for Institutional University Co-operation (IUC).

\section{References}

1. Papanicolaou, G.: A new procedure for staining vaginal smears. Science 95, 438-439 (1942)

2. Pantanowitz, L., Hornish, M., Goulart, R.: The impact of digital imaging in the field of cytopathology. Cytojournal 6(1), 6-15 (2010)

3. Ricketts, I., Banda-Gamboa, H., Cairns, A., Hussein, K.: Automatic classification of cervical cells-using the frequency domain. In: IEEE Colloquium on Applications of Image Processing in Mass Health Screening, IET, p. 9 (2002)

4. Walker, R., Jackway, P.: Statistical geometric features extensions for cytological texture analysis. In: Proceedings of 13th International Conference on Pattern Recognition, vol. 2, pp. 790-794 (1996)

5. Lezoray, O., Cardot, H.: Cooperation of color pixel classification schemes and color watershed: a study for microscopic images. IEEE transactions on Image Processing 11, 783-789 (2002)

6. Bak, E., Najarian, K., Brockway, J.: Efficient segmentation framework of cell images in noise environments. In: 26th IEEE Annual International Conference on Engineering in Medicine and Biology Society (IEMBS 2004), vol. 1, pp. 1802-1805 (2005) 
7. Beucher, S.: Watershed, hierarchical segmentation and waterfall algorithm. Mathematical morphology and its applications to image processing, 69-76 (1994)

8. Roerdink, J., Meijster, A.: The watershed transform: Definitions, algorithms and parallelization strategies. Mathematical morphology 187 (2000)

9. DiZenzo, S.: A note on the gradient of a multi-image. Comput. Vision, Graphics. Image Proc. 33(1), 116-125 (1986)

10. Geerinck, T., Sahli, H., Henderickx, D., Vanhamel, I., Enescu, V.: Modeling attention and perceptual grouping to salient objects. In: Paletta, L., Tsotsos, J.K. (eds.) WAPCV 2008. Lecture Notes in Computer Science(LNAI), vol. 5395, pp. 166-182. Springer, Heidelberg (2009)

11. Lucchi, A., Smith, K., Achanta, R., Lepetit, V., Fua, P.: A Fully Automated Approach to Segmentation of Irregularly Shaped Cellular Structures in EM Images. In: Medical Image Computing and Computer-Assisted Intervention-MICCAI 2010, pp. 463-471 (2010)

12. Calderero, F., Marques, F.: General region merging approaches based on information theory statistical measures. In: 15th IEEE International Conference on Image Processing, ICIP 2008, pp. 3016-3019 (2008)

13. Platt, J.C.: Probabilistic outputs for support vector machines and comparison to regularized likelihood methods. In: Advances in large margin classifiers, pp. 61-74 (1999)

14. Cristianini, N., Shawe-Taylor, J.: Introduction to Support Vector Machines and other kernel-based learning methods. Cambridge University Press, Cambridge (2000)

15. Borsotti, M., Campadelli, P., Schettini, R.: Quantitative evaluation of color image segmentation results. Pattern Recognition Letters 19(8), 741-747 (1998)

16. Jennrich, R., Sampson, P.: Stepwise discriminant analysis. In: Mathematical methods for digital computers, pp. 339-358 (1960)

17. Canu, S., Grandvalet, Y., Rakotomamonjy, A.: SVM and Kernel Methods MATLAB Toolbox. Perception de Syst émes et Information, INSA de Rouen, France (2003)

18. Joshi, M.V.: On evaluating performance of classifiers for rare classes. In: Proceedings of the IEEE International Conference on Data Mining ICDM 2002, p. 641. IEEE Computer Society, Washington (2002)

19. Vanhamel, I., Mihai, C., Sahli, H., Katartzis, A., Pratikakis, I.: Scale Selection for Compact Scale-Space Representation of Vector-Valued Images. International Journal of Computer Vision 84(2), 194-204 (2009) 